\title{
Effective Truncation of a Student's $t$-Distribution by Truncation of the Chi Distribution in a Chi-Normal Mixture
}

\author{
Daniel T. Cassidy \\ Department of Engineering Physics, McMaster University, Hamilton, Canada \\ Email: cassidy@mcmaster.ca
}

Received September 25, 2012; revised October 27, 2012; accepted November 10, 2012

\begin{abstract}
A Student's $t$-distribution is obtained from a weighted average over the standard deviation of a normal distribution, $\sigma$, when $1 / \sigma$ is distributed as chi. Left truncation at $q$ of the chi distribution in the mixing integral leads to an effectively truncated Student's $t$-distribution with tails that decay as $\exp \left(-q^{2} t^{2} / 2\right)$. The effect of truncation of the chi distribution in a chi-normal mixture is investigated and expressions for the pdf, the variance, and the kurtosis of the $t$-like distribution that arises from the mixture of a left-truncated chi and a normal distribution are given for selected degrees of freedom $\leq 5$. This work has value in pricing financial assets, in understanding the Student's $t$-distribution, in statistical inference, and in analysis of data.
\end{abstract}

Keywords: Asset Pricing; Student's $t$-Distribution; Cauchy; Truncation; Moments; Kurtosis

\section{Introduction}

A Student's $t$-distribution is used for statistical inference for small sample sizes [1,2]. Nadarajah [3] states that the Student's $t$-distribution is the second most popular distribution, second in popularity only to the normal distribution.

In addition to statistical inference, the Student's $t$-distribution has application in finance, wherein the $t$-distribution is found to fit the distribution of the logarithms of daily returns better than a normal distribution or indeed better than most any other distribution [4-9]. The number of degrees of freedom, $v$, is found by least squares fitting to historical returns to be around 3 for daily returns of the DJIA and S\&P 500 indices [9], with increasing $v$ for $n$-day returns, $n>1$.

The Student's $t$-distribution also has application wherever a Cauchy (Lorentzian) distribution is employed, since a Student's $t$-distribution with one degree of freedom is a Cauchy distribution.

The Student's $t$-distribution offers support from $-\infty$ to $+\infty$ and has tails that decrease as $t^{v+1}$ for $t^{2}>>v$. This causes problems in finance as integrals needed to price financial instruments diverge for the logarithm of returns distributed as a Student's $t$-distribution [9-11] and the frequency of occurrence of the logarithms of daily returns is fit well by Student's $t$-distribution.
Truncation, capping, and modification of the $t$-distribution have been put forth as means to deal with the divergence [8,9,12]. Moriconi [8] multiplied the Student's $t$-distribution by an $\exp \left(-t^{2}\right)$ to keep integrals finite in pricing options. Lim et al. [12] used a generalized $t$-distribution [13], which has a multiplicative term of $\exp \left(-t^{4}\right)$. Lim et al. [12] reported that the generalized $t$-distribution fit currency options better than any other probability density function (pdf) that was used. The results presented here provide a physical basis for understanding the origin of a multiplicative factor.

Praetz [4] and Gerig et al. [7] pointed out that a Student's $t$-distribution is obtained from a mixture of a normal distribution with a standard deviation $\sigma$ that is distributed as an inverse chi distribution. If the support for the inverse chi distribution is taken to be zero to infinity, then a Student's $t$-distribution is obtained from the mixture. For convenience, in this paper the reciprocal of $\sigma$ is denoted as $a, a=1 / \sigma$, and $a$ is distributed as chi, $\chi(a ; v, \beta)$ :

$$
\chi(a ; v, \beta) \mathrm{d} a=\frac{\left(v \beta^{2}\right)^{(v-1) / 2} a^{v-1} \mathrm{e}^{-v \beta^{2} a^{2} / 2}}{\Gamma(v / 2) 2^{v / 2-1}} \beta \sqrt{v} \mathrm{~d} a .
$$

Using chi as defined above and a normal distribution with zero mean and standard deviation of $\sigma=1 / a$, the mixing integral, when evaluated from $a=0$ to $a=+\infty$ 
yields a Student's $t$-distribution

$$
\begin{aligned}
f_{\mathbf{t}}(t ; v, \beta) & =\int_{0}^{\infty} \frac{a \mathrm{e}^{-a^{2} t^{2} / 2}}{\sqrt{2 \pi}} \chi(a ; v, \beta) \mathrm{d} a \\
& =\frac{\Gamma((v+1) / 2) v^{v / 2} \beta^{v}}{\sqrt{\pi} \Gamma(v / 2)\left(t^{2}+v \beta^{2}\right)^{(v+1) / 2}}
\end{aligned}
$$

with a mean of zero, $v$ degrees of freedom, and a scale parameter of $\beta$. The parameters for the chi distribution were chosen to yield a Student's $t$-distribution, $f_{t}(t ; v, \beta)$, with a mean of zero, $v$ degrees of freedom, and a scale parameter of $\beta$.

If a chi distribution for the reciprocal of $\sigma$ is left truncated, then the result of the mixing integral with the left-truncated chi distribution is a $t$-like distribution that has exponentially decaying tails. This result is demonstrated for $v=3$. The integrals involved can be evaluated analytically for odd $v$. Small values of $v$ are of interest.

The contribution to a $v=3$ Student's $t$-distribution, $f_{t}(t ; v=3, \beta)$, from values in the left-hand wing of the chi distribution for $a \leq q$ is given by

$$
\begin{aligned}
& l(t ; v=3, \beta, q)=\int_{0}^{q} \frac{a \mathrm{e}^{-a^{2} t^{2} / 2}}{\sqrt{2 \pi}} \chi(a ; v=3, \beta) \mathrm{d} a \\
& =3 \frac{\sqrt{3} \beta^{3}\left(2 \mathrm{e}^{q^{2}\left(t^{2}+3 \beta^{2}\right) / 2}-3 \beta^{2} q^{2}-2-q^{2} t^{2}\right) \mathrm{e}^{-q^{2}\left(t^{2}+3 \beta^{2}\right) / 2}}{\pi\left(t^{2}+3 \beta^{2}\right)^{2}},
\end{aligned}
$$

whereas the contribution from values in the right-hand wing of the chi distribution for $a>q$ is given by

$$
\begin{aligned}
& r(t ; v=3, \beta, q)=\int_{q}^{\infty} \frac{a \mathrm{e}^{-a^{2} t^{2} / 2}}{\sqrt{2 \pi}} \chi(a ; v=3, \beta) \mathrm{d} a \\
& =3 \frac{\sqrt{3} \beta^{3}\left(3 \beta^{2} q^{2}+2+q^{2} t^{2}\right) \mathrm{e}^{-q^{2}\left(t^{2}+3 \beta^{2}\right) / 2}}{\pi\left(t^{2}+3 \beta^{2}\right)^{2}} .
\end{aligned}
$$

It is interesting to note that the sum of the contributions from Equations (3) and (4) form a full Student's $t$-distribution (in this case the mixing integral has been written as the sum of two exhaustive and exclusive regions: $a \leq q$ and $a>q$ ), and that the right-hand contribution has an exponentially decaying tail. As a result, any pdf that does not include the left-hand contribution from the chi distribution for the reciprocal of the standard deviation in a chi-normal mixture will have tails that decay as $\exp \left(-q^{2} t^{2} / 2\right)$.

Figure 1 is a plot of a Student's $t$-distribution with $v=3$ degrees of freedom and the contributions to the $t$-distribution from the right-hand wing of the chi distribution and from the left-hand wing of the chi dis-

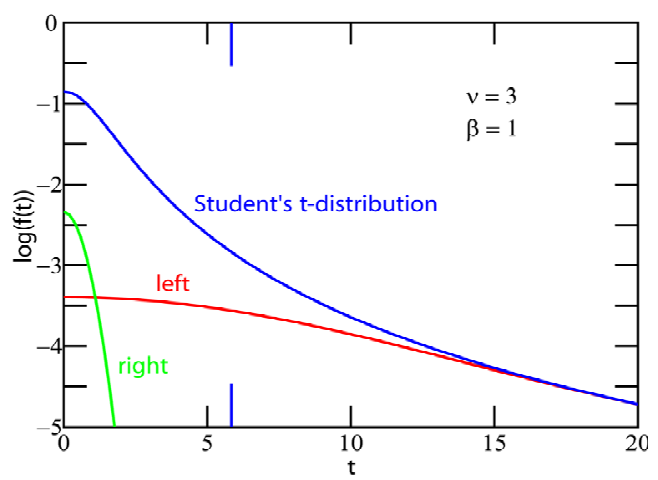

Figure 1. Semi-log plots of a Student's $t$-distribution with $v$ $=3$ and scale parameter $\beta=1$ and of the contributions to the $t$-distribution from the left- and right-hand wings of the chi distribution in the chi-normal mixture that produces the Student's $t$-distribution.

tribution. Figure 1 clearly shows that large values of the standard deviation $\sigma$ in the mixture give rise to the fat tails of the $t$-distribution. The large values of the standard deviation occur for small values of the reciprocal of the standard deviation. For Figure 1, the wing of the chi distribution was truncated for an area of 0.01 in the wing. The small values of the standard deviation (large reciprocal; right wing) make a negligible contribution to the value of the Student's $t$-distribution. The long tic mark at $t=5.841$ marks the point where the area in each tail of the Student's $t$-distribution equals 0.005 , for a total area of 0.01 .

The slowly decaying power tails of the $t$-distribution with increasing $t$ results in the divergence of moments and of integrals required to price financial instruments that are based on a $\log$ Student's $t$-distribution. The results presented in Figure 1 are interesting on two levels. One level is intrinsic interest in understanding the Student's $t$-distribution and the other level is use. The results show the origins of the slowly decaying power tails of Student's $t$-distributions and suggest an approach to deal with the divergence of moments and integrals based on the $t$-distribution.

Left truncation of the chi distribution for the reciprocal of the standard deviation to allow for only physically possible values of the standard deviation $\sigma$ will impart exponentially decaying tails to the resulting $t$-like distribution from the chi-normal mixture. It is unlikely that $0 \leq \sigma \leq \infty$. A value of $\sigma=0$ implies no variability and a value of $\sigma=\infty$ implies infinite variability.

In the following sections, expressions for the probability density function (pdf), the variance, and the kurtosis are given for several small values of $v$ for $t$-like distributions that are obtained by left truncation of a chinormal mixture. In addition, the first several terms for power series expansions of the variance and kurtosis 
are given. These power series demonstrate the effect of the left truncation on the moments. Since the power series are valid only for small amounts of truncation, Figure 2 is presented. This figure shows the magnitude of the parameters involved for a left truncation of the chi distribution, $\chi(a ; v, \beta)$. After Figure 2, some general definitions are given.

Figure 2 plots the cumulative density function (CDF) $F(x)=\int_{0}^{x} \chi(a ; v, \beta=1) \mathrm{d} a$ for small values of the independent variable $x$ for the chi distribution in the mixing integral that yields a Student's $t$-distribution with $v=$ $1,2,3,5$ and 9 degrees of freedom. The long tic marks give the values $(q=0.0125,0.10,0.196,0.333,0.482)$ for which the CDF $F(q)=0.01$. Figure 2 allows an appreciation of the differences amongst the chi distributions for different degrees of freedom and of the magnitudes of the numbers involved.

The probability that $x>q, P_{\chi}(x>q ; v, \beta)$, is needed to normalize properly a truncated chi distribution. A left-truncated chi distribution $\chi(a ; v, \beta)$ is zero for values $a \leq q$ :

$$
P_{\chi}(\boldsymbol{x}>q ; v, \beta)=\int_{q}^{\infty} \chi(x ; v, \beta) \mathrm{d} x .
$$

$f(t ; v, \beta, q)$ is the pdf for a mixture of a left-truncated chi and normal distribution. If $q=0$ then $f(t ; v, \beta, q)$ would be a Student's $t$-distribution with $v$ degrees of freedom and scale parameter $\beta$. An explicit expression for $f(t ; v, \beta, q)$ is

$$
f(t ; v, \beta, q)=\int_{q}^{\infty} \frac{a \mathrm{e}^{-a^{2} t^{2} / 2}}{\sqrt{2 \pi}} \frac{\chi(a ; v, \beta)}{P_{\chi}(\boldsymbol{x}>q ; v, \beta)} \mathrm{d} a .
$$

$V(v, \beta, q)$ is the variance of the pdf $f(t ; v, \beta, q)$,

$$
V(v, \beta, q)=\int_{-\infty}^{\infty} t^{2} f(t ; v, \beta, q) \mathrm{d} t,
$$

and $K(v, \beta, q)$ is the kurtosis of the pdf $f(t ; v, \beta, q)$,

$$
K(v, \beta, q)=\frac{1}{V(v, \beta, q)^{2}} \int_{-\infty}^{\infty} t^{4} f(t ; v, \beta, q) \mathrm{d} t .
$$

This paper provides information on the effective truncation of a Student's $t$-like distribution when the chi distribution for the reciprocal of the standard deviation is left-truncated. It is shown that the tails of the effectively truncated $t$-distribution go as $\exp \left(-q^{2} t^{2} / 2\right)$. The contribution $r(t ; v, \beta, q)$ to an odd $v$ Student's $t$-distribution from values of $a>q$ for a chi-normal mixture, i.e., Equation (4) evaluated for odd $v$, is given by (see Equation (9) below).

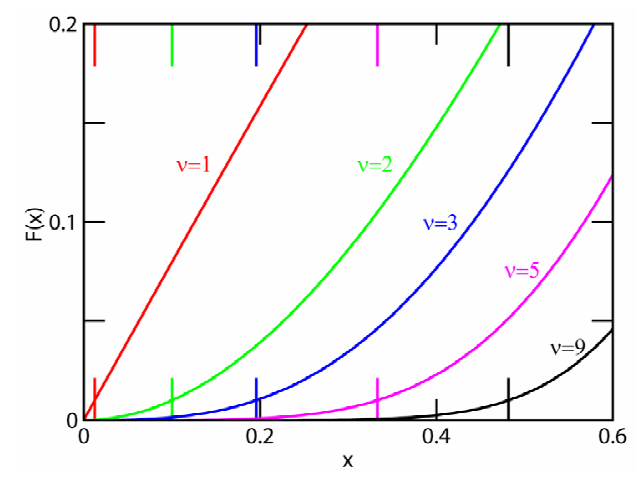

Figure 2. Plot of $\boldsymbol{F}(x)=\int_{0}^{x} \chi(a ; v, \beta=1) \mathrm{d} a$ for small values of $x$ and for $v=1,2,3,5$ and 9. The long tic marks on the abscissa mark the values for which the area to the left equals 1\%, i.e., $P_{x}(x<q ; v, \beta=1)=F(q)=0.01$.

The expression, which decreases with $t$ as $\exp \left(-q^{2} t^{2} / 2\right)$, was obtained from an examination of the expressions for $r(t ; v, \beta, q)$ for $v \leq 11$ and by comparison with the general case for odd $v$. Clearly, left truncation of the chi distribution for the reciprocal of the standard deviation in a chi-normal mixture removes the fat tails of the Student's $t$-distribution.

\section{Results}

Expressions for some of the low $v$, effectively truncated Student's $t$-distributions and moments are given in the following subsections. For a Student's $t$-distribution the variance equals $\beta^{2} v /(v-2)$ and exists only for $v>2$. The kurtosis equals $3(v-2) /(v-4)$ and exists only for $v>4$. For effectively truncated Student's $t$-distributions (i.e., truncation of the large values of the standard deviation in the chi mixing distribution), the moments exist for all $v$.

\section{1. $v=1$ (Cauchy or Lorentzian Distribution)}

The pdf for a mixture of a left-truncated chi distribution for $v=1$ and $a=1 / \sigma$ and a normal distribution is

$$
\begin{aligned}
& f(t ; v=1, \beta, q) \\
& =\frac{\beta \mathrm{e}^{-q^{2}\left(t^{2}+\beta^{2}\right) / 2}}{\left(t^{2}+\beta^{2}\right) \pi(1-\operatorname{erf}(\beta q / \sqrt{2}))} .
\end{aligned}
$$

The tails of the pdf decrease as $\exp \left(-q^{2} t^{2} / 2\right)$ for non-zero $q$.

$$
\frac{\beta^{v} \sqrt{v^{v}} \mathrm{e}^{-q^{2}\left(t^{2}+v \beta^{2}\right) / 2}\left(\left(\frac{v-1}{2}\right) ! \sqrt{2^{v-1}}+\sum_{i=0}^{(v-1) / 2}\left(\left(t^{2}+v \beta^{2}\right)^{\frac{v-1-2 i}{2}} \prod_{j=0}^{i-1}(v-1-2 j)\right)\right)}{(v-2) ! ! \pi\left(t^{2}+v \beta^{2}\right)^{(v+1) / 2}} .
$$


The first moment for $t>0$ exists for the effectively truncated $v=1 \quad t$-distribution and is given by

$$
\begin{aligned}
\int_{0}^{\infty} t f(t ; v=1, \beta, q) \mathrm{d} t & =\frac{\beta \operatorname{Ei}\left(1, \beta^{2} q^{2} / 2\right)}{2 \pi(1-\operatorname{erf}(\beta q / \sqrt{2}))} \approx-\frac{\beta\left(\gamma+\ln \left(\beta^{2} q^{2} / 2\right)\right)}{2 \pi}-\frac{\beta\left(\gamma+\ln \left(\beta^{2} q^{2} / 2\right)\right)}{\sqrt{2} \pi^{3 / 2}} \beta q \\
& -\frac{\beta\left(-\pi+4 \gamma+4 \ln \left(\beta^{2} q^{2} / 2\right)\right)}{4 \pi^{2}} \beta^{2} q^{2}+O\left(\beta^{3} q^{3}\right)
\end{aligned}
$$

where $\gamma=0.5722 \cdots$ is Euler's contant and $\mathrm{Ei}()$ is the exponential integral. The series expansion is valid for $\beta q<<1$. The series expansion shows a logarithmic divergence as $\beta q$ approaches zero, as expected for a Cauchy distribution.

The $v=1$ variance is given by

$$
\begin{aligned}
V(v=1, \beta, q) & =\frac{\beta \mathrm{e}^{-\beta^{2} q^{2} / 2}\left(-\beta \pi \mathrm{e}^{\beta^{2} q^{2} / 2} q+\beta \pi \mathrm{e}^{\beta^{2} q^{2} / 2} \operatorname{erf}(q \beta / \sqrt{2}) q+\sqrt{2 \pi}\right)}{q \pi(1-\operatorname{erf}(q \beta / \sqrt{2}))} \\
& \approx \frac{\sqrt{2} \beta^{2}}{\sqrt{\pi} \beta q}+\frac{(-\pi+2) \beta^{2}}{\pi}-\frac{\beta^{3} \sqrt{2}(\pi-4)}{2 \pi^{3 / 2}} q+O\left(\beta^{2} q^{2}\right),
\end{aligned}
$$

is proportional to $\beta^{2}$, remains finite for $q>0$, and diverges as $1 / q$ as $q$ approaches zero.

An exact expression for the $v=1$ kurtosis can be found, but the expression is long and cumbersome. The series expansion

$$
K(v=1, \beta, q) \approx \frac{\sqrt{2 \pi}}{2 q \beta}+\pi-1+3 \sqrt{2 \pi}(\pi-3) \beta q+O\left(\beta^{2} q^{2}\right)
$$

shows that the $v=1$ kurtosis is proportional to $1 / q$ and hence stays finite for $q>0$. Both the variance and the kurtosis are not defined for a Cauchy distribution, i.e., for a Student's $t$-distribution with $v=1$ and a region of support from $-\infty$ to $+\infty$.

\section{2. $v=2$}

The pdf, $f(t ; v=2, \beta, q)$, for a mixture of a left-truncated chi distribution for $v=2$ and $a=1 / \sigma$ with a normal pdf is

$$
\begin{aligned}
& \frac{\beta^{2}\left(q \sqrt{2} \sqrt{t^{2}+2 \beta^{2}}+\sqrt{\pi}\left(1-\operatorname{erf}\left(q \sqrt{\left(t^{2}+2 \beta^{2}\right) / 2}\right)\right) \mathrm{e}^{q^{2}\left(t^{2}+2 \beta^{2}\right) / 2}\right) \mathrm{e}^{-q^{2} t^{2} / 2}}{\sqrt{\pi}\left(t^{2}+2 \beta^{2}\right)^{3 / 2}} \\
& \approx \beta^{2}\left(t^{2}+2 \beta^{2}\right)^{-\frac{3}{2}}-\frac{1}{2}\left(t^{2}-\left(t^{2}+2 \beta^{2}\right)\right)\left(t^{2}+2 \beta^{2}\right)^{-\frac{3}{2}} \beta^{2} q^{2}+O\left(\beta^{3} q^{3}\right) .
\end{aligned}
$$

The first term in the series expansion is equal to $f_{\mathrm{t}}(t ; v=2, \beta)$.

$f(t ; v=2, \beta, q)$ has tails that decrease as $\exp \left(-q^{2} t^{2} / 2\right)$ for non-zero $q$, since $\sqrt{\pi}(1-\operatorname{erf}(x)) \approx \exp \left(-x^{2}\right) / x$ for large $x$. For large $q t$,

$$
f(t ; v=2, \beta, q) \approx\left(\frac{\beta^{2} q \sqrt{2}}{\sqrt{\pi} t^{2}}+O\left(t^{-3}\right)\right) \mathrm{e}^{-q^{2} t^{2} / 2}
$$

Simple, analytic expression for the moments for even values of $v$ could not be found.

\section{3. $v=3$}

The $v=3$ pdf, $f_{t}(t ; v=3, \beta)$, equals

$$
\frac{3 \mathrm{e}^{-q^{2} t^{2} / 2}\left(3 \beta^{2} q^{2}+2+q^{2} t^{2}\right) \beta^{3} \sqrt{3}}{\sqrt{\pi}\left(t^{2}+3 \beta^{2}\right)^{2}\left(\sqrt{\pi} \mathrm{e}^{3 / 2 \beta^{2} q^{2}}+\sqrt{6} \beta q-\sqrt{\pi} \operatorname{erf}(\sqrt{6} \beta q / 2) \mathrm{e}^{3 / 2 \beta^{2} q^{2}}\right)}
$$


and has tails that decrease as $\exp \left(-q^{2} t^{2} / 2\right)$ for non-zero $q$.

The $v=3$ variance is given by

$$
\begin{aligned}
& V(v=3, \beta, q) \\
& =\frac{3 \pi \beta^{2}(1-\operatorname{erf}(1 / 2 \sqrt{6} \beta q))}{\sqrt{\pi} \beta q \sqrt{6} \mathrm{e}^{-3 / 2 \beta^{2} q^{2}}+\pi(1-\operatorname{erf}(1 / 2 \sqrt{6} \beta q))} \\
& \approx 3 \beta^{2}-\frac{3 \sqrt{6}}{\sqrt{\pi}} \beta^{3} q+\frac{9 \sqrt{6}}{2 \sqrt{\pi}} \beta^{5} q^{3}+O\left(\beta^{4} q^{4}\right)
\end{aligned}
$$

and approaches $3 \beta^{2}$ as $q$ approaches zero. This is expected since the variance for a Student's $t$-distribution exists for $v>2$ and equals $\beta^{2} v /(v-2)$ or $3 \beta^{2}$ for $v=3$.

A series expansion for the $v=3$ kurtosis (the exact expression is long and cumbersome) is

$$
\begin{aligned}
K(v=3, \beta, q) \approx & \frac{\sqrt{6}}{\beta q \sqrt{\pi}}+3 \frac{4-\pi}{\pi}+\frac{9 \sqrt{6}(4-\pi)}{2 \pi^{3 / 2}} \beta q \\
& -48 \frac{(\pi-3)}{\pi^{2}} \beta^{2} q^{2}+O\left(\beta^{3} q^{3}\right),
\end{aligned}
$$

and diverges as $1 / q$ as $q$ approaches zero. The kurtosis for a Student's $t$-distribution is defined only for $v>4$.

\section{4. $v=5$}

The series expansion for the $v=5$ variance shows a weak dependence on the left truncation. To lowest order in $q$, the $v=5$ variance has a cubic dependence on $q$. The curves of Figure 2 show that the chi distribution has decreasing area in the left for small $q$ as $v$ increases.

$$
V(v=5, \beta, q) \approx \frac{5}{3} \beta^{2}-\frac{25}{9} \frac{\beta^{2} \sqrt{10}}{\sqrt{\pi}} \beta^{3} q^{3}+O\left(\beta^{5} q^{5}\right)
$$
by

The series expansions for the $v=5$ kurtosis is given

$$
\begin{aligned}
& K(v=5, \beta, q) \approx 9-9 \frac{\sqrt{10}}{\sqrt{\pi}} \beta q \\
& +\frac{75}{2} \frac{\sqrt{10}}{\sqrt{\pi}} \beta^{3} q^{3}-\frac{300}{\pi} \beta^{4} q^{4}+O\left(\beta^{5} q^{5}\right) .
\end{aligned}
$$

The $v=5$ kurtosis is finite for $q=0$. The series expansion is a linear decrease in $q$ for small $q$.

For $v=7$, the first two terms of the alternating series for the expansion of the kurtosis are $5\left(1-4.93 \beta^{3} q^{3}\right)$. For $v>5$ the kurtosis is not impacted by a truncation for small $\beta q$.

\section{Application}

Application of the effectively truncated Student's $t$-distribution to pricing a European call option is given in this section.

The value of a European call option at the time of expiration, $C_{T}$, is the expectation of the maximum value of $\left\{S_{T}-K_{T}, 0\right\}, C_{T}=E\left\{\left(S_{T}-K_{T}\right)^{+}\right\}$, where $S_{T}$ is the price of a stock at time $T, K_{T}$ is the strike price at time $T, T$ is the time when the option expires, and $E\{\}$ is the expectation operator [9]. It is not necessary to solve a partial differential equation to find the price of an option [9-11]. The desired quantity $C_{0}$, which is the value of the option at time $t=0$, is obtained from the expected time value of money. If $r(t)$ is the risk free rate as a function of time $t$, then $C_{0}=C_{T} \times \exp (-r \times T)$ when the risk free rate is assumed to be time independent. This is a standard assumption in the derivation of the Black-Scholes formula.

Let $S_{T}=A_{T} \exp \left(\sigma_{T} \xi\right)$ be the value of the stock at time $T$ where $\xi$ is a random variable, and where both $A_{T}$ and the volatility $\sigma_{T}$ do not depend on the random variable $\xi$.

In terms of the pdf for $\xi, f_{\xi}(\xi)$, the value of the option at time $T$ is

$$
C_{T}=\int_{\ln \left(\frac{K_{T}}{A_{T}}\right) / \sigma_{T}}^{\infty}\left(A_{T} \exp \left(\sigma_{T} \xi\right)-K_{T}\right) f_{\xi}(\xi) \mathrm{d} \xi
$$

The value of $A_{T}$ is determined by the requirements that the process be fair (i.e., the process is a martingale) and that the development in time of the price include the time value of money [9]. This requires that $E\left\{A_{T} \exp \left(\sigma_{T} \xi\right)\right\}=\exp (r T) S_{0}$ where $S_{0}$ is the value of the stock at time 0 .

If $\xi$ is normally distributed, then $S_{T}$ follows a $\log$ normal distribution, $A_{T}=S_{0} \exp (r T) \exp \left(-\sigma_{T}^{2} / 2\right)$, and the price for the option is given by the Black-Scholes formula.

If $\xi$ follows a Student's $t$-distribution, then $C_{T}$ is infinite. The exponential growth of $S_{T}$ with $\xi$ dominates the $\xi^{-(v+1)}$ tails of the Student's distribution, Equation (2). However, if an effectively truncated Student's $t$-distribution is used, then the tails of the effectively truncated $t$-distribution diminish with $\xi$ as $\exp \left(-q^{2} \xi^{2} / 2\right)$, and the value of the European call option remains finite. Both the effectively truncated Student's $t$-distribution and the normal distribution have a multiplicative $\exp \left(-\xi^{2} / 2\right)$ factor that dominates the exponential growth of $S_{T}$ for large $\xi$. In general, the integral to price the European call option must be evaluated numerically. Equation (21) is perhaps the simplest manner in which to write the cost of the call option.

The Student's $t$-distribution is found (over the subinterval of the infinite region of support where returns are observed) to fit the distribution of the logarithms of daily returns better than a normal distribution or indeed better than most any other distribution [4-9]. It would be 
prudent to price options using a distribution that matches the data. The effectively truncated $t$-distribution that is described in this paper is a distribution that matches the observed data, as demonstrated in Figure 3.

Adaptive bins widths were used to generate the frequency of occurrence data for Figure 3. The bin width was increased until at least 5 counts were obtained in the bin. The normal distribution fits the data well only in the neighbourhood of $t=0$ and does not fit the fat tails of the data. The quality of the fit of a Student's $t$-distribution to the 15491 data points that constitute the frequency of occurrence of ranges of values for the logarithms of the daily returns is remarkably good over all the returns, including the infrequent, low-probability events in the tails.

A fit of a Student's $t$-distribution to the logarithm of the daily returns over the period of January 1950 to 27 July 2011 for the S\&P 500 Index gives $v=3.3 \pm 0.2$ and a scale parameter $\beta=(6.06 \pm 0.3) \times 10^{-3}$. The data show a kurtosis of 25 and a maximum 22-day volatility of 0.0573 . The approximation for the kurtosis for a $v=3$ effectively truncated $t$-distribution equal to 25 , $K(v=3, \beta, q)=25$, is solved for $\beta q=0.057$. The chi distribution in the mixing integral is, assuming that $v=3$, truncated for volatilities $>0.1=\beta / 0.057$ to obtain the same kurtosis as the data. This level of truncation is roughly twice the maximum 22-day volatility of 0.0573 that was obtained from the daily returns. Note that the kurtosis exists only for $v>4$ for Student's $t$ distributions. In contrast, the kurtosis is defined for $v<4$ for an effectively truncated $t$-distribution, in agreement with the data.

The area in the left wing for truncation of the chi distribution at $\beta q=0.057$ to match the kurtosis of the observed daily returns of 25 is $2.6 \times 10^{-4}$. This level of truncation of the chi distribution has a minor effect on the effectively truncated Student's $t$-distribution for $|t|<20$, as can be observed in Figure 4. This is consistent with the data; all 15491 data points lie in the interval -0.205 to +0.116 . When the scale parameter $\beta=(6.06 \pm 0.3) \times 10^{-3}$ is taken into account, the data lie in the region $-33.7<t<19.1$ The truncation to match the kurtosis is not severe (c.f. Figure 4), but the truncation is sufficient to keep finite the integrals required to price options with a log Student's $t$-distribution.

\section{Conclusions}

A Student's $t$-distribution arises from an averaging over the standard deviation of a normal distribution when the reciprocal of the standard deviation is distributed as chi. The Student's $t$-distribution offers support over $-\infty$ to $+\infty$. The slowly decaying power tails and infinite support region mean some moments for the Student's $t$-distribution do not exist. This divergence of integrals

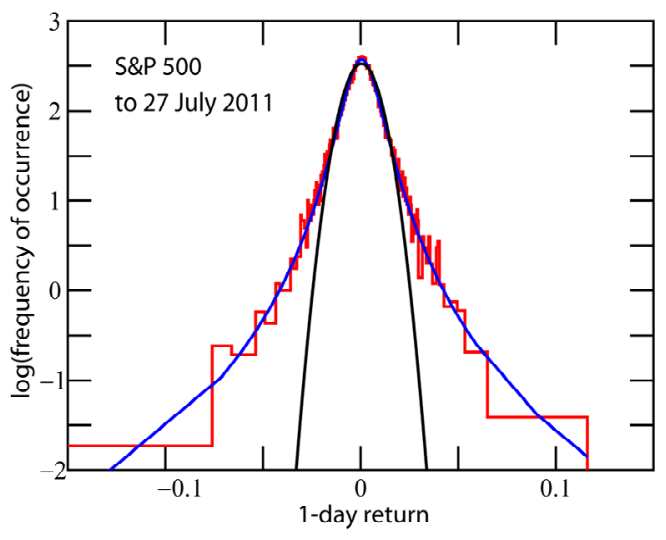

Figure 3. Plots of the best fit Student's $t$-distribution and normal distribution to the frequency of occurrence of the logarithms of daily returns for the S\&P 500 index. The Student's $t$-distribution fits the data well.

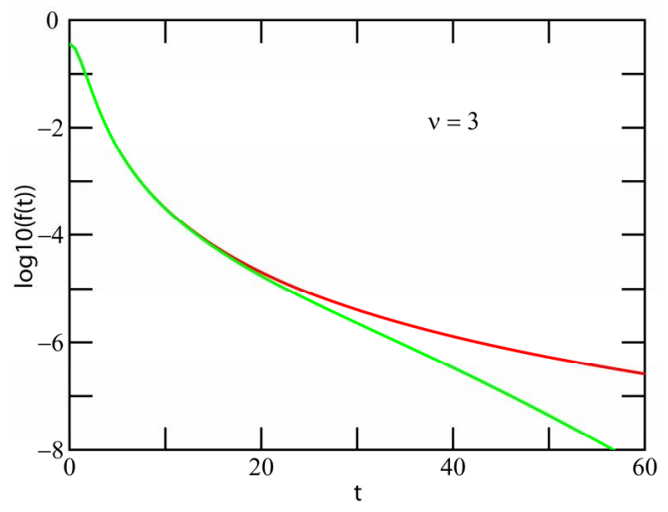

Figure 4. Plot of a $v=3$ Student's $t$-distribution (red) and an effectively truncated $v=3$ Student's $t$-distribution (green). The truncation was chosen to match the observed kurtosis of the daily returns for the S\&P 500 index over the period of January 1950 to 27 July 2011.

can cause problems, particularly in the pricing of options where the logarithm of returns is distributed as a Student's $t$-distribution. One approach to deal with the divergence is to truncate the Student's $t$-distribution [9]. Data typically fit well to the central region of the $t$-distribution and are nonexistent far from the central region of the Student's $t$-distribution. Other approaches are to multiply the $t$-distribution by an envelope function that reduces the impact of the tails [8] or to use a generalized form that includes an envelope function that reduces the impact of the tails [12]. The approach presented here is to left-truncate a chi distribution for the reciprocal of the standard deviation. The chi distribution is used in a mixing integral with a normal distribution to yield an effectively truncated Student's $t$-distribution. Left truncation of the chi distribution at $q$ yields tails for the effectively truncated Student's $t$-distribution that decay with increasing $t$ as $\exp \left(-q^{2} t^{2} / 2\right)$. The approach adopted here 
supports the envelope multiplication of Moriconi [8] by showing that the envelope modification is similar in effect to a left truncation of a chi distribution for the reciprocal of the standard deviation, and then mixing the truncated chi distribution with a normal distribution of the same standard deviation.

Expressions or power series expressions for the pdf, variance, and kurtosis for several low number of degrees of freedom, effectively truncated Student's $t$-distributions are given. These expressions demonstrate the exponential tails of the effectively truncated $t$-distribution and show that the variance and kurtosis remain remain finite for the effectively truncated distributions. In addition, it is shown that it is the large values of the standard deviation in the mixing integral that give rise to the fat tails of the Student's $t$-distribution. The small values of the standard deviation in the mixing integral do not contribute to the tails of the Student's $t$-distribution and only weakly contribute to the core of the Student's $t$-distribution.

The effective truncation of the Student's $t$-distribution means that integrals required to price financial instruments such as European call options remain finite. This permits pricing with a distribution, the log Student's $t$-distribution, that describes well returns for stocks.

Simple expressions were not found for the effectively truncated $t$-distributions with even numbers of degrees of freedom. Given the importance of the Student's $t$-distribution in the description of returns and data in general, it would be helpful to find accurate approximations that smoothly approach the expressions for the odd numbers of degrees of freedom. These approximations could then be used to model data where it is unphysical to assume support over $-\infty$ to $+\infty$. The $\exp \left(-q^{2} t^{2} / 2\right)$ envelope modification of Moriconi [8] might be a sufficiently good approximation for all degrees of freedom. It is shown that the envelope modification of Moriconi is functionally equivalent to a truncation of large values of the volatility (standard deviation) in the chi distribution of the chi-normal mixing integral that leads to a Student's $t$-distribution.

\section{Acknowledgements}

This work was funded by the Natural Science and Engineering Research Council (NSERC) Canada.

\section{REFERENCES}

[1] Student, "The Probable Error of a Mean," Biometrika,
Vol. 6, No. 1, 1908, pp. 1-25.

[2] S. L. Zabell, "On Student's 1908 Article 'The Probable Error of a Mean'," Journal of the American Statistical Association, Vol. 103, No. 481, 2008, pp. 1-7. doi: $10.1198 / 016214508000000030$

[3] S. Nadarajah, "Explicit Expressions for Moments of $t$ Order Statistic," Comptes Rendus de l'Académie des Sciences-Series I, Vol. 345, No. 9, 2007, pp. 523-526. doi:10.1016/j.crma.2007.10.027

[4] P. D. Praetz, "The Distribution of Share Price Changes," The Journal of Business, Vol. 45, No. 1, 1972, pp. 49-55. doi: $10.1086 / 295425$

[5] R. C. Blattberg and N. Gonedes, "A Comparison of the Stable and Student Distributions as Statistical Models for Stock Prices," The Journal of Business, Vol. 47, No. 2, 1974, pp. 244-280. doi:10.1086/295634

[6] E. Platen and R. Sidorowicz, "Empirical Evidence on Student- $t$ Log-Returns of Diversified World Stock Indices," Research Paper 194, Quantitative Finance Research Centre, University of Technology, Sydney, 2007.

http://www.business.uts.edu.au/qfrc/research/research_pa pers/rp194.pdf

[7] A. Gerig, J. Vicente and M. Fuentes, "Model for Non-Gaussian Intraday Stock Returns," Physical Review E, Vol. 80, No. 6, 2009, pp. 1-4. doi:10.1103/PhysRevE.80.065102

[8] L. Moriconi, "Delta Hedged Option Valuation with Underlying Non-Gaussian Returns," Physica A, Vol. 380, No. 1, 2007, pp. 343-350. doi:10.1016/j.physa.2007.01.018

[9] D. T. Cassidy, M. J. Hamp and R. Ouyed, "Pricing European Options with a Log Student's $t$-Distribution: A Gosset Formula," Physica A, Vol. 389, No. 24, 2010, pp. 57365748. doi:10.1016/j.physa.2010.08.037

[10] J.-P. Bouchaud and D. Sornette, "The Black-Scholes Option Pricing Problem in Mathematical Finance: Generalization and Extensions for a Large Class of Stochastic Processes," Journal de Physique I (France), Vol. 4, No. 6, 1994, pp. 863-881. doi:10.1051/jp1:1994233

[11] J. L. McCauley, G. H. Gunaratne and K. E. Bassler, "Martingale Option Pricing," Physica A, Vol. 380, No. 1, 2007, pp. 351-356. doi:10.1016/j.physa.2007.02.038

[12] G. C. Lim, G. M. Martin and V. L. Martin, "Pricing Currency Options in the Presence of Time-Varying Volatility and Non-Normalities," Journal of Multinational Financial Management, Vol. 16, No. 3, 2006, pp. 291-314. doi:10.1016/j.mulfin.2005.08.004

[13] J. N. Lye and V. L. Martin, "Robust Estimation, NonNormalities and Generalized Exponential Distributions," Journal of the American Statistical Association, Vol. 88, No. 421, 1993, pp. 261-267. doi:10.2307/2290721 